\title{
Atomic force microscopy visualises a hydrophobic meshwork in the central channel of the nuclear pore
}

\author{
Armin Kramer • Ivan Liashkovich • Yvonne Ludwig • \\ Victor Shahin
}

Received: 27 September 2007 / Accepted: 9 November 2007 / Published online: 4 December 2007

(C) Springer-Verlag 2007

\begin{abstract}
Nuclear pore complexes (NPCs) mediate and control the transport of virtually all material between the cytosol and the nucleus. It is, therefore, unsurprising that they have long taken centre stage in physiology. A precise understanding of the NPC structure and function that remain to be thoroughly investigated yet is, thus, of crucial importance. The NPC can mediate transport both actively and passively. It remains to be clarified, however, whether transport of small molecules and macromolecules proceeds through the same route in the NPC. Furthermore, it has been shown that surface hydrophobicity represents a major sorting criterion for the active transport through NPCs. Transport factors like importin $\beta$, which exhibit a rather large surface hydrophobicity, bind to their cargo and are believed to interact with a supposedly hydrophobic meshwork that is assumed to reside in the central channel of the NPC but has not yet been visualised. This interaction is presumed to lead to a partial breakdown of the meshwork, thereby, permitting the transport-cargo complexes to pass through. In this study, by using the nano-imaging approach, atomic force microscopy, we visualised under near-physiological conditions, for the first time, the presence of a hydrophobic meshwork in the NPC central channel. Furthermore, our data lend strong support for the existence of two segregated transport routes in the NPC.
\end{abstract}

Keywords Fluorescence - Ion transport .

Membrane transport $\cdot$ Microscopy $\cdot$ Xenopus laevis

A. Kramer $(\square) \cdot$ I. Liashkovich $\cdot$ Y. Ludwig $\cdot$ V. Shahin $(\square)$ Institute of Physiology II, University of Münster,

Robert-Koch Str. 27 b,

48149 Münster, Germany

e-mail: kramercom@gmail.com

e-mail: shahin@uni-muenster.de

\section{Introduction}

The nuclear envelope separates the nuclear from the cytoplasmic compartment. Exchange of material between both compartments is mediated and controlled by nuclear pore complexes (NPCs) that are at regular distances embedded into junctions formed between the inner and the outer membranes of the nuclear envelope. NPCs are large protein assemblies consisting of multiple copies of up to 30 different proteins designated nucleoporins (nups) [1]. Translocation of material across NPCs proceeds by means of passive or active transport. Whereas molecules up to $9 \mathrm{~nm}$ [2] in diameter can traverse the NPC by passive diffusion, larger molecules need to be transported actively. The latter transport is receptor-mediated and restricted to molecules bearing specific localisation signals, which are recognised by transport factors such as importin $\alpha-\beta[3,4]$. The transport factors first recognise/bind the transport cargo and then jointly target the NPC through which they are ultimately translocated. Pante and Kann [5] have shown that the cutoff for active transport across NPCs is at 39-nm molecule diameter. There is increasing functional evidence that a highly selective hydrophobic barrier in the central channel of NPCs retains inert molecules from passing through and that this barrier is presumably formed by a meshwork of protein filaments that protrude into the central channel of the NPC [6-9]. Interestingly, despite being the subject of a large number of studies, the presence of such filaments in the NPC central channel has lacked structural evidence up to now. Basis of this meshwork are weak hydrophobic interactions of nup domains, which are rich in phenylalanine- and glycine-(FG)-repeat motifs. Transport factors have been shown to exhibit a relatively high surface hydrophobicity, which enables them to interrupt the hydrophobic meshwork and to shuttle cargo between the 
nucleoplasm and the cytoplasm. The mesh size of the barrier has been calculated to be about $3 \mathrm{~nm}$ [10]. This value does not match the limit for passive diffusion cutoff of $9 \mathrm{~nm}$. Moreover, there is increasing evidence that the receptor-mediated transport and passive diffusion of ions and small macromolecules occurs via peripheral routes in the NPC, presumably, the so-called peripheral channels, whose presence has been initially postulated by electron microscopy in 1992 [11]. Support for the existence of peripheral channels in the NPC comes from later studies investigating the NPC both from structural and functional aspects using permeability investigating approaches and the nano-imaging technique atomic force microscopy (AFM), respectively [12-15]. AFM not only yields extraordinary high-resolution images [16] but can also be used as a nano-mechanical tool for studying numerous properties of the imaged sample such as hydrophobicity [17, 18]. In this study, by using AFM under physiological conditions, we provide, for the first time, structural evidence for the presence of a hydrophobic meshwork in the NPC central channel. Furthermore, by combing AFM with other established methods, we localise the cytoplasmic entrance of the peripheral channels in the NPC.

\section{Experimental procedures}

\section{Isolation of cell nuclei}

Female Xenopus laevis were anaesthetised with $0.1 \%$ ethyl $m$-aminobenzoate methanesulfonate (Serva, Heidelberg, Germany), and their ovaries were removed. Oocytes were dissected from ovary clusters and stored in modified Ringer's solution $(87 \mathrm{mM} \mathrm{NaCl}, 6.3 \mathrm{mM} \mathrm{KCl}, 1 \mathrm{mM}$ $\mathrm{MgCl}_{2}, 1.5 \mathrm{mM} \mathrm{CaCl}$, $10 \mathrm{mM}$ 4-2-hydroxyethyl-1piperazineethanesulfonic acid (HEPES), $100 \mathrm{U} / 100 \mu \mathrm{g}$ penicillin/streptomycin; $\mathrm{pH}$ 7.4) before use. For isolation of the cell nuclei, the oocytes were transferred into nuclear isolation medium (NIM) composed of (in millimolar) $90 \mathrm{KCl}, 26 \mathrm{NaCl}, 5.6 \mathrm{MgCl}_{2}$ (corresponding to a free $\mathrm{Mg}^{2+}$ concentration of $\left.2 \mathrm{mM}\right), 1.1$ ethylene glycol bis(2aminoethyl ether)- $N, N, N^{\prime} N^{\prime}$-tetraacetic acid and 10 HEPES, and titrated to $\mathrm{pH}$ 7.4. We added $1.5 \%$ polyvinylpyrrolidone (PVP, $\mathrm{Mr}=40,000$; Sigma) to compensate for the lack of macromolecules in the NIM, mimicking the intact cytosol. The presence of PVP is crucial to preventing the swelling (more than $100 \%$ in the absence of PVP) of total nuclear volume that occurs instantaneously after isolation in pure electrolyte solution.

Preparation of nuclear envelopes

After placing the oocytes in NIM, nuclei were isolated manually by piercing the oocyte with two pincers.
Individual intact nuclei were picked up with a Pasteur pipette and transferred to a glass coverslip placed under a stereomicroscope. The chromatin was then carefully removed using sharp needles, and the nuclear envelope was spread on poly-l-lysine-coated (Sigma, St. Louis, MO, USA) glass, with the nucleoplasmic side facing downwards. Finally, the specimens were fixed using $2 \%$ glutaraldehyde and $1 \%$ paraformaldehyde for $1 \mathrm{~h}$ at room temperature, washed three times with NIM and investigated with AFM without any drying procedure.

Nuclear hourglass technique

The technical aspects of the method and its application to isolated cell nuclei have previously been described in detail $[12,19]$. In brief, the method uses a tapered glass tube that narrows in the middle to two thirds the diameter of the nucleus. A current of up to $1 \mathrm{~mA}$ is injected via two $\mathrm{Ag} / \mathrm{AgCl}$ electrodes through either end of the glass tube. The voltage drops across the cell nucleus are measured with two conventional microelectrodes whose tips are placed near the narrow part of the capillary opposite each other so as to measure the electrical resistance of the fluid column between them. Because current and voltage are measured simultaneously, the resistance can be calculated online and monitored during the measurements. The nucleus is sucked into the narrow part of the capillary by gentle fluid movement. Thus, the current flows through the nucleus. The resulting increase in total electrical resistance indicates the specific electrical resistance of the cell nucleus. The nuclear resistance after incubation with antibodies or importin $\beta$ 45-462 was measured using alternating square-wave current pulses of $15 \mu \mathrm{A}$.

Incubation of cell nuclei with NPC antibodies and importin ß 45-462

Cell nuclei were incubated with $1 \mu \mathrm{M}$ of the respective antibody or $0.5 \mu \mathrm{M}$ of importin $\beta \quad 45-462$ at room temperature in NIM with $1.5 \%$ PVP. Subsequently, the nuclei were washed for $10 \mathrm{~min}$ in NIM with $1.5 \% \mathrm{PVP}$, and their electrical resistance was measured using the NHT. Alternatively, for the immunostaining, nuclei were incubated for $1 \mathrm{~h}$ with $0.1 \mu \mathrm{M}$ of the secondary antibody (Cy3 labelled anti-rabbit-IgG) and then washed in NIM with $1.5 \%$ PVP. Nuclear envelopes were next dissected and spread out on poly-l-lysine-coated glass.

Detection of antibodies on the nuclear envelope surface by immunofluorescence

Fluorescence measurements of nuclear envelope preparations were performed using an inverted microscope (Axiovert 100, 
Zeiss, Germany) equipped with a cooled charge-coupled device camera and the imaging software MetaMorph (Visitron Systems, Puchheim, Germany).

\section{Expression of importin $\beta$ 45-462}

The expression plasmid for importin $\beta$ 45-462 was a kind gift from Prof. Dirk Görlich/ZMB Heidelberg. Importin $\beta$ 45-462 was expressed and purified as described previously [20]. Briefly, the expression plasmid (pQE60, Qiagen, Hilden, Germany), containing the coding sequence of human importin $\beta 45-462$ and an N-terminal his-tag, was used to transform Escherichia coli (BLR/Rep4). Expression was at $18^{\circ} \mathrm{C}$ for $3 \mathrm{~h}$. After centrifugation, the bacterial pellet was resuspended in $50 \mathrm{mM}$ Tris $-\mathrm{HCl}$, $\mathrm{pH} 7.5,20 \mathrm{mM}$ imidazole, $200 \mathrm{mM} \mathrm{NaCl}$ and $5 \mathrm{mM}$ mercaptoethanol, and lysis was performed by sonication. The lysate was cleared by centrifugation at $14,000 \times \mathrm{g}$ for $30 \mathrm{~min}$ and loaded to a column containing nickel agarose. The column was washed with the same buffer and eluded with $300 \mathrm{mM}$ imidazole. The buffer was exchanged for $50 \mathrm{mM}$ Tris- $\mathrm{HCl}$ and $250 \mathrm{mM}$ sucrose.

\section{Atomic force microscopy}

The application of AFM to nuclear envelopes has been described in detail elsewhere [21]. We used a MultimodeTM atomic force microscope (with a NanoScope V controller; Veeco, Mannheim, Germany) equipped with an optical microscope, a video camera, and a monitor to visualise the nuclear envelope and the AFM tip on its stage. We used standard V-shaped 115- $\mu \mathrm{m}$-long silicon nitride cantilevers with a spring constant of $0.32 \mathrm{~N} / \mathrm{m}$ and pyramidal tips with an estimated tip diameter of $10 \mathrm{~nm}$ (DNP-S tips, Veeco). The images were recorded in tapping mode with 512 lines per screen and at a scan rate of $1.5 \mathrm{~Hz}$. The forces applied during the scanning procedure were minimised by retracting the AFM tip until it lost contact with the sample surface and reengaging the tip at an amplitude set point (i.e. force value) as close as possible to the lift-off value. Although the AFM tip physically interacts with the nuclear envelope, the repeatability of results from multiple scans indicated that the preparation was not damaged. Moreover, scanning at low forces ( $3 \mathrm{nN}$ or less) left no visible marks in the preparation.

Tapping mode and phase imaging

In tapping mode, the cantilever is oscillated at a frequency near its resonance. As the probe lightly taps the surface of the sample, the amplitude of oscillation is reduced, and the AFM uses this change in amplitude to track the surface topography. Eventual mechanical damage to the sample is reduced in tapping mode as lateral and shear forces are virtually eliminated. In addition to its amplitude, the probe motion in tapping mode can be characterised by detecting the phase difference between the exciting signal and the cantilever oscillation. The measured phase shift is very sensitive to several surface properties such as elasticity, adhesion, hydrophobicity/hydrophylicity and surface charges $[17,18]$. The phase difference or phase shift, which is recorded simultaneously with the changes in the amplitude, can be used to create an image in which the degree of phase shift is colour coded. Therefore, combining tapping mode with phase imaging produces a high-resolution image of the scanned sample and maps its surface properties in one go. Figure 1 illustrates the principle of phase imaging on a flat surface with different material properties. Whilst measuring rough biological samples, however, it needs to be emphasised that not only differences in the material properties of the sample can change the phase shift but also differences in the height. The phase image, therefore, reflects both changes in height and changes in the material properties of the sample. Hence, it is crucial to compare the phase image with the corresponding height image to obtain information about the material properties of a sample.

\section{Antibodies}

The anti-Nup358 antibody [22] was generously provided by Mary Dasso (Laboratory of Gene Regulation and Development, NICHD/NIH, Bethesda, USA). The antibody against the short C-terminal cytoplasmic domain of gp210 [23] was a kind gift by Katherine L. Wilson (Johns Hopkins School of Medicine, Baltimore, USA), and the antibody against Nup88 [24] was kindly provided by Maarten Fornerod (Netherlands Cancer Institute, Amsterdam, The
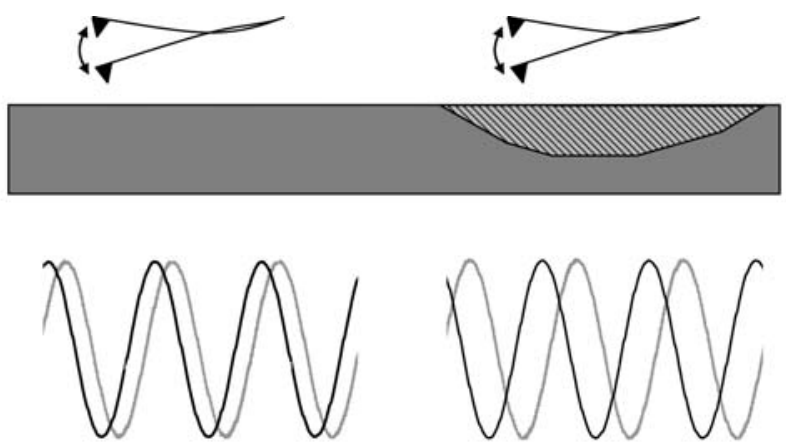

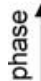

Fig. 1 Principle of phase imaging. The AFM cantilever oscillates whilst the tip lightly taps the surface of the sample. Between the exciting signal (solid line) and the cantilever oscillation (dashed line) is a slight difference, the phase shift. As the AFM tip encounters a surface with different material properties (right-hand side), this phase shift can change. The different phase shifts can be used to create an image of the surface investigated (not shown) 
Netherlands). Cy3-labeled (anti-rabbit-IgG) secondary antibodies were purchased from Dianova, Hamburg, Germany.

\section{Results}

Visualisation of a meshwork in the NPC central channel using phase imaging

We applied AFM to provide structural evidence for the presence of a hydrophobic meshwork residing in the NPC central channel. Figure 2 shows the height image and the corresponding phase image, as well as exemplified crosssections of the cytoplasmic face of a dissected nuclear envelope. At a first glance, both images seem fairly identical, although differing in contrast. Closer inspection of the two images, however, shows clearly that the NPC central channels in the phase image are generally occupied by a structure, fairly reminding on a meshwork, which is hardly visible in the height image, however. In other words, the height image failed to detect this meshwork, meaning that the latter most likely reveals material properties (such as degree of hydrophobicity) different from those of the rest of the NPC.

The meshwork in the NPC central channel is rather hydrophobic

We addressed the question as to whether the meshwork in the NPC central channel has only rendered visible in the phase image because of different degree of hydrophobicity compared with the rest of the NPC. For this reason, a mutant of importin $\beta$ (importin $\beta$ 45-462) was applied, which binds irreversibly to the central channel and, thereby, effectively obstructs it. Importin $\beta$ belongs to the most hydrophobic soluble proteins in a eukaryotic cell [8]. We incubated oocyte nuclei with the mutant protein and prepared nuclear envelopes for investigation with AFM. Figure 3 shows a height and a phase image of NPCs whose central channels are obstructed by the importin $\beta$ mutant. Again, on comparing the two images with each other, it stands out that the mutant protein is markedly more
Fig. 2 Height image (a) and the simultaneously recorded phase image (b) of the cytosolic side of a nuclear envelope with exemplified cross-sections on the right-hand side
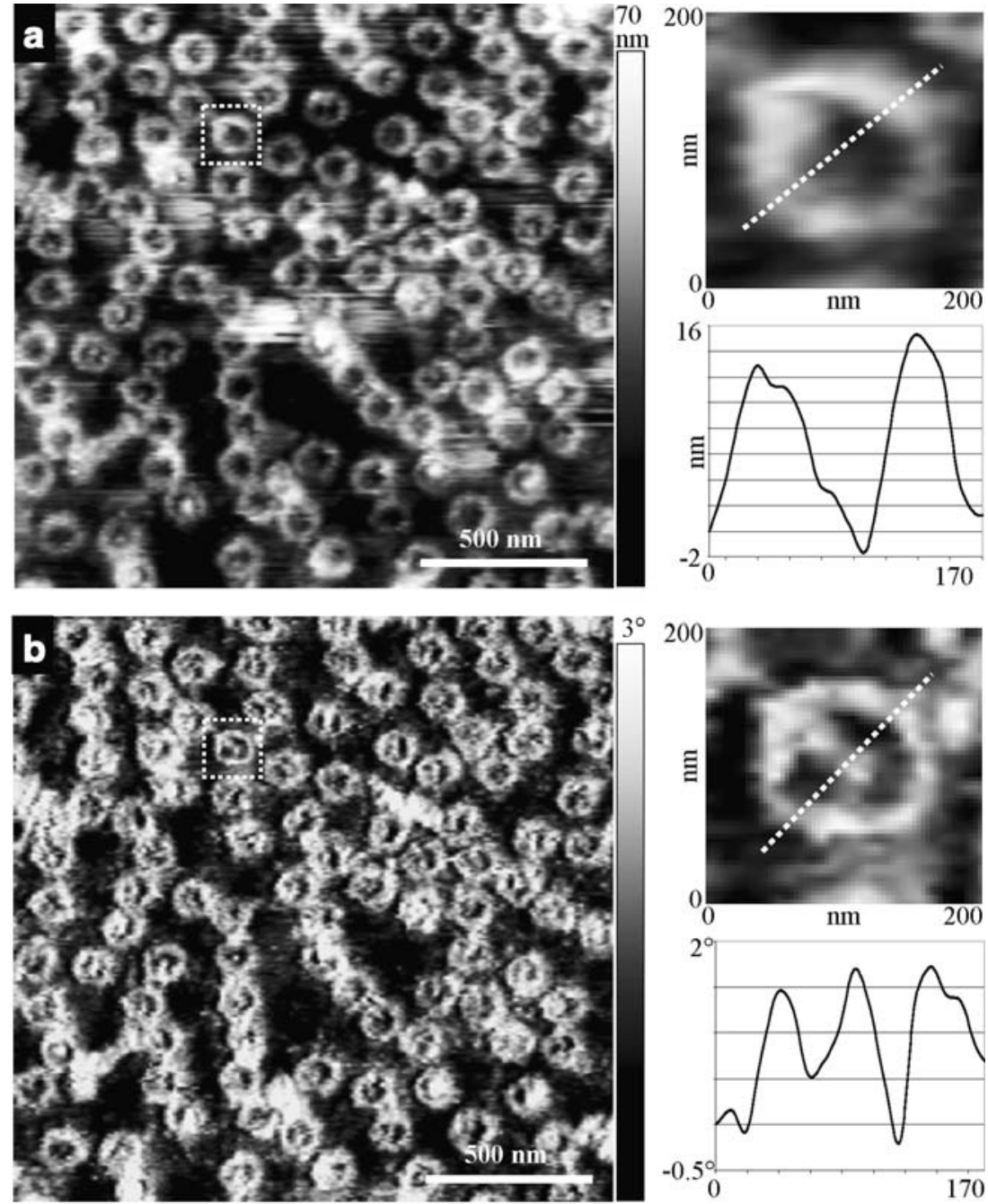
Fig. 3 Height image (a) and phase image (b) of nuclear pores plugged with importin $\beta$ 45-462 with exemplified cross-sections on the right-hand side
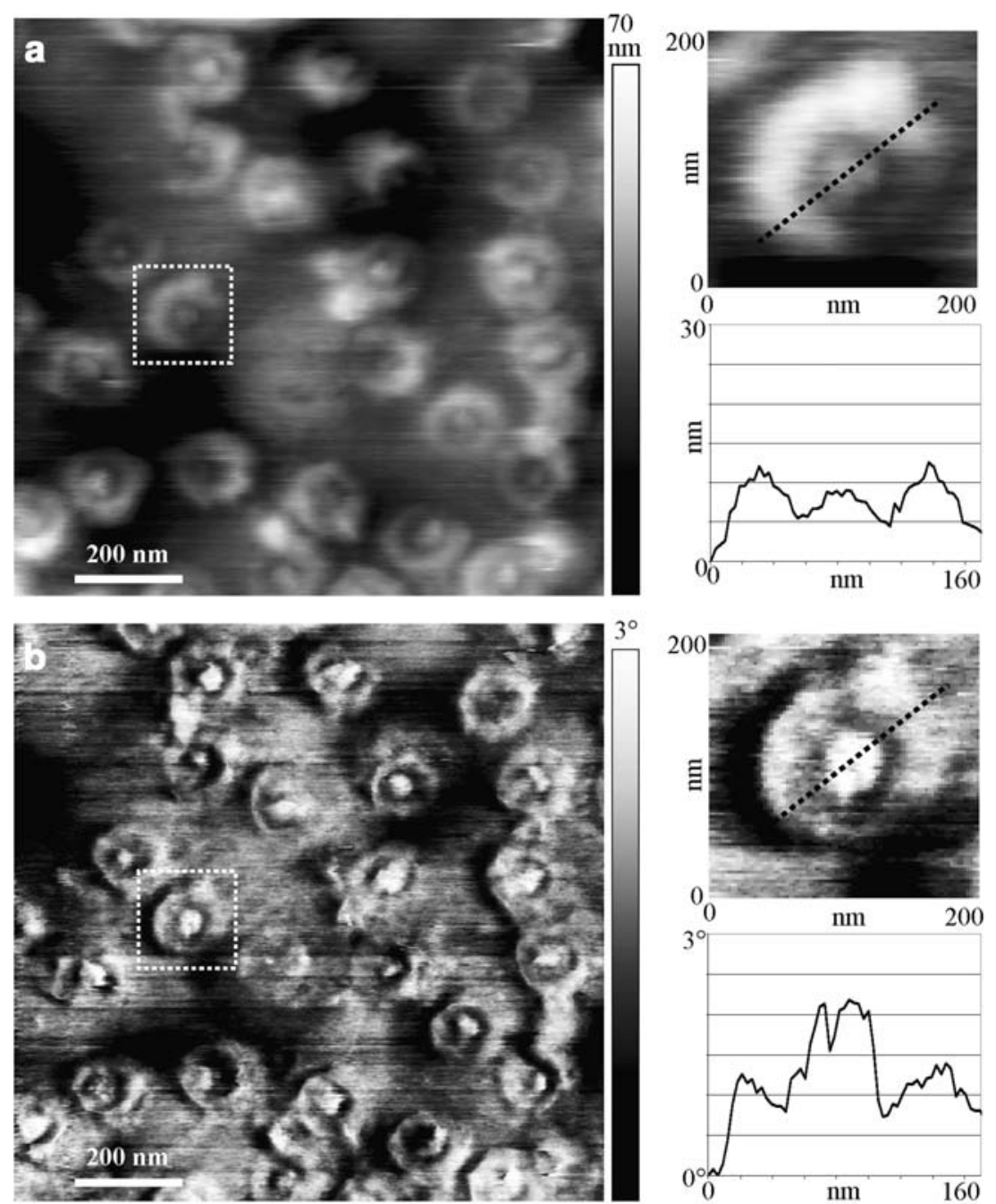

contrasted in the phase than in the height image, in which pore rim and the importin $\beta$ plug are more or less at the same level of height. The relatively hydrophobic importin $\beta$ mutant exhibits a change in phase shift compared to the pore rim, which has the same orientation and a comparable offset as the change seen between the central channel and the rim in Fig. 2.

Localisation of ion permeable sites at the NPC segregated from the NPC central channel

The importin $\beta$ mutant was further employed to investigate whether the observed central channel obstruction would lead to an increase in electrical resistance of the nuclear membrane. The electrical resistance, or rather the electrical conductivity, which is the reciprocal value of the resistance, is a direct measure of the ion permeability. Moreover, we incubated oocyte nuclei with antibodies against three peripheral proteins (or domains) of the NPC to test whether this would lead to an increase in electrical resistance. Figure 4 depicts schematically the targets of the three antibodies at the
NPC. The binding of the antibodies was confirmed by immunofluorescence (Fig. 5). For the electrical measurements, we used the nuclear hourglass technique (NHT), by means of which the resistance of whole nuclei can be recorded. Figure 6 displays the outcome of the measurements. Amongst the three antibodies against peripheral domains of NPCs, only the antibody raised against Nup358 showed a significant effect. The two other antibodies and the importin $\beta$ mutant show no significant effect on the electrical resistance.

\section{Discussion}

NPCs have long taken centre stage in physiology and have, therefore, been the subject of a great number of studies investigating them from various perspectives such as structurally and functionally. As physiologists, we are particularly interested in combining structural with functional investigations under physiological conditions, and this blatantly cries out for applying AFM, which has long 
Fig. 4 Schematic of the binding sites of the three antibodies at the NPC. Nup358 forms a part of the cytoplasmic ring and the filaments. Nup88 is likewise a constituent of the cytoplasmic ring. Nup210 is a central nup against its short cytosolic

C-terminal domain the antibody is directed (arrow). In the middle of the pore, the hydrophobic meshwork of FG-repeat domains is indicated. $C P$ Cytoplasm, NP nucleoplasm, $O M$ outer membrane, $I M$ inner membrane

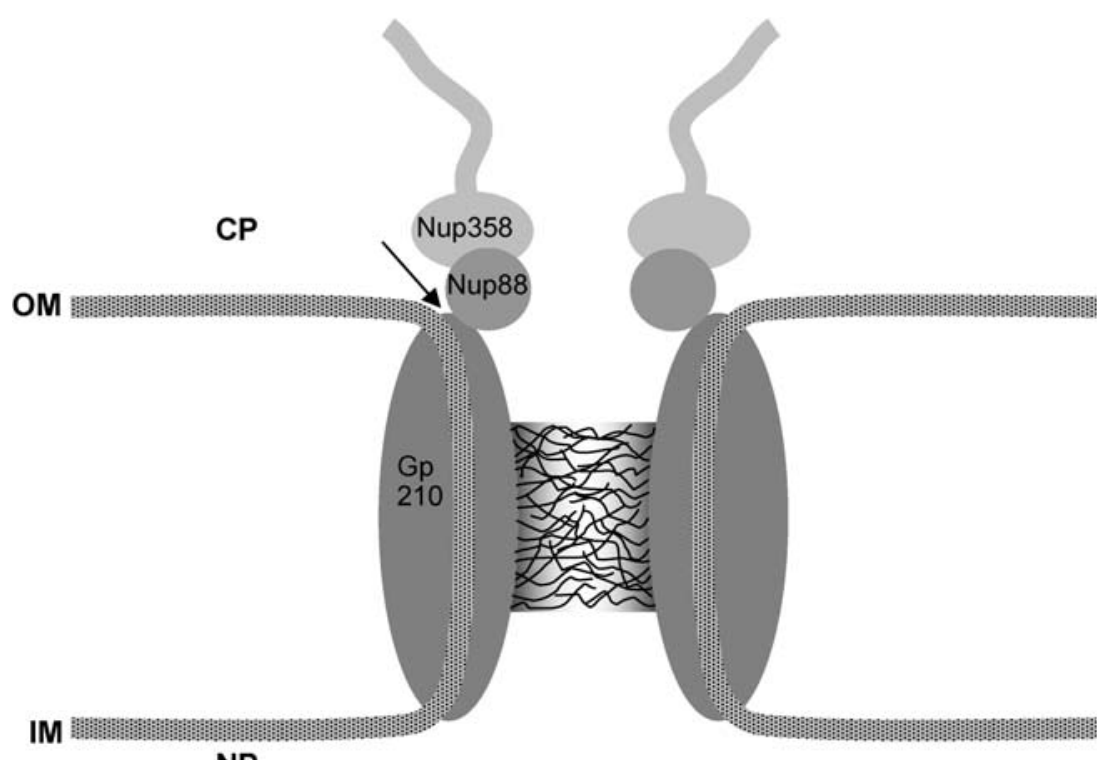

NP proved to be the technique for such purposes. In this study, AFM was applied to shed light on some aspects concerning the structure and function of the NPC. Thereby, we addressed the long-standing question as to whether an individual NPC features two separate transport pathways, one for large macromolecules and another for small molecules. Furthermore, we aimed to visualise a meshwork, hydrophobic in nature and residing in the NPC central channel.
The NPC harbours a hydrophobic meshwork residing in its central channel and mediates transport of small molecules and macromolecules through separate routes

One of the ultimate goals of this work was to provide structural evidence for the presence of a hydrophobic meshwork residing in the NPC central channel by using AFM. By recording height and phase images simultaneously, we visualised NPCs and mapped some of their surface
Fig. 5 Immunofluorescence images of nuclear envelopes with the cytoplasmic side facing up. a Membrane incubated with anti-Nup88 and Cy3-labeled anti-rabbit-IgG. b Membrane incubated with anti-Nup358 and Cy3-labeled anti-rabbit-IgG. c Membrane incubated with anti-gp210 and Cy3-labeled anti-rabbit-IgG. d Membrane incubated with Cy3-labeled antirabbit-IgG alone (control)
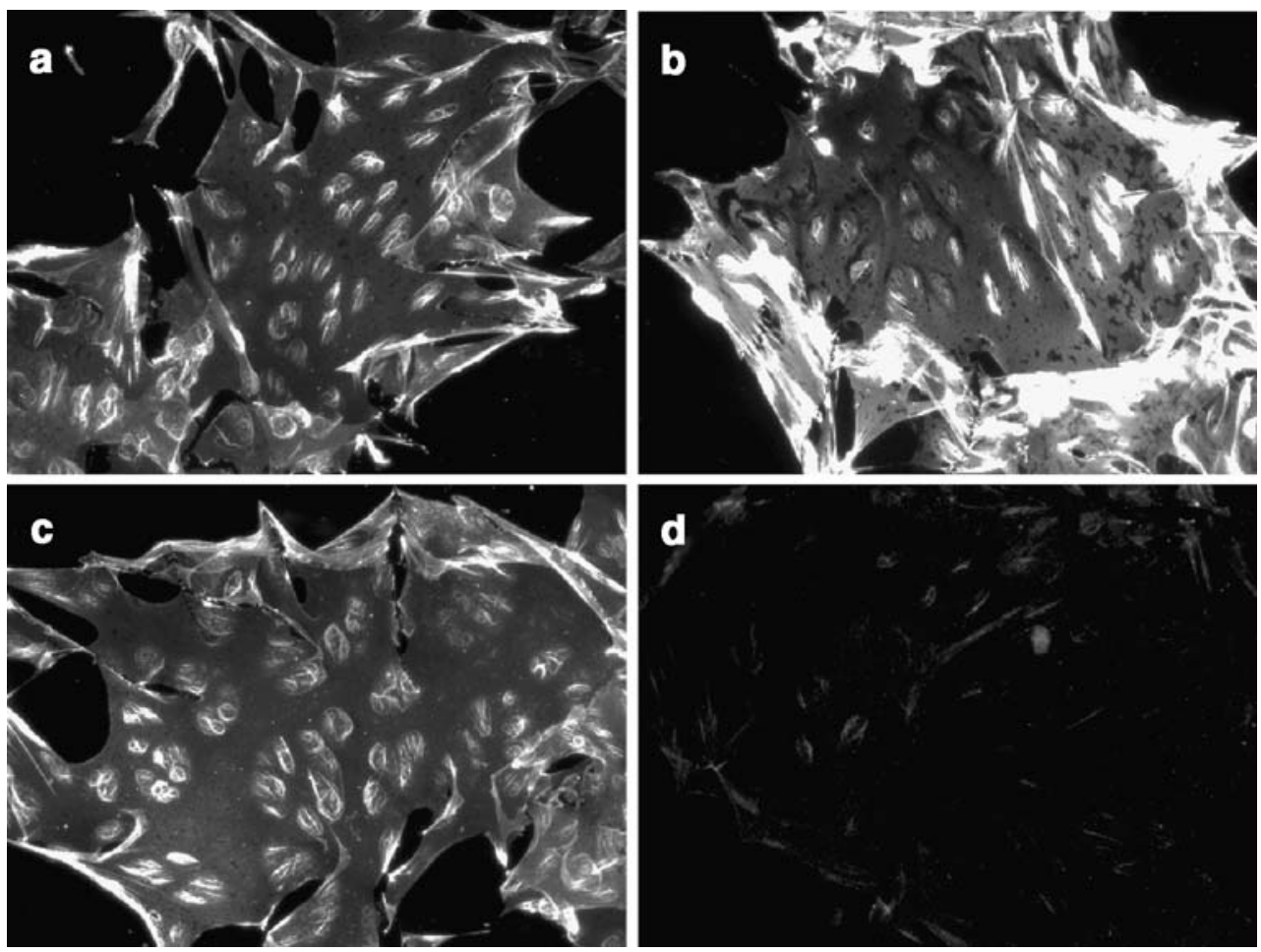


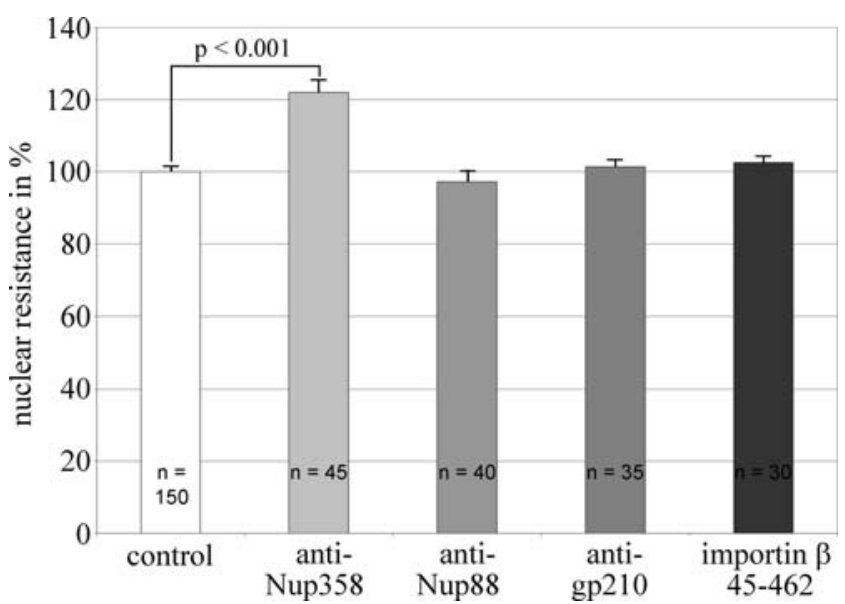

Fig. 6 Measurement of the nuclear resistance upon incubation with different antibodies or importin $\beta 45-462$ in NIM. Controls were incubated in NIM without antibody. Statistical significant difference exists only between control and anti-Nup358 incubation (Student's $t$ test, $p<0.001)$. Error bars are given as standard error of the mean (SEM). $n$ Number of nuclei

properties such as surface hydrophobicity, which stood in the focus of our attention indeed. On comparison of the simultaneously recorded height and phase images of NPCs with each other, the following observation was made. The phase image has rendered a meshwork-like structure residing in the NPC central channel. The corresponding height image, however, fairly failed to render the same structure visible. With respect to its shape, this structure was presumed to be the meshwork that has been dealt with previously, and the conclusion was drawn that the phase image not only yielded information about the height but also about the surface properties of the detected NPCs. In other words, the surface properties of the meshwork in the NPC central channel were fairly different from those of the rest of the NPC. The question was still to be addressed, however, as to what different surface properties have led to the detection of the meshwork in the phase but not in the height image. More precisely and with regard to the assumed hydrophobicity of the meshwork $[6,8,9]$, we attempted to answer the question as to whether the meshwork has only rendered visible in the phase image because of the different degree of its surface hydrophobicity when compared with the rest of the NPC. The ultimate goal of this attempt was to provide, for the first time, structural evidence for the existence of a meshwork residing in the NPC central channel and hydrophobic in nature. As mentioned previously, the presence of such a hydrophobic meshwork has repeatedly been assumed but never really proved under conditions, such as those used in this study, namely, 'poking' into the NPC central channel right where the meshwork is assumed to reside. Analysis of importin $\beta$ plugged pores gave rise to the assumption that the meshwork visualised in the NPC central channel is hydrophobic in nature; the relatively hydrophobic importin $\beta$ mutant exhibits a change in phase shift compared to the pore rim, which has the same orientation and a comparable offset as the change seen between the central channel and the rim in Fig. 2. Indeed, we are well aware of the fact that some surface properties other than hydrophobicity might also have affected the phase shift mentioned above to some extent. However, further support for the hydrophobicity being the major cause of the above-mentioned phase shift is provided considering the following. The oxide sharpened $\mathrm{Si}_{3} \mathrm{~N}_{4}$ tips used in these experiments were hydrophilic [25], and therefore, interactions between the tip and the hydrophobic meshwork were expected to be rather repulsive. It has been shown in theory and in practice that repulsive tip-sample interactions cause a positive phase shift or phase advance, whereas attractive interactions result in a negative phase shift or phase lag $[17,18,26]$. The positive peak seen in the central channel in the phase image of Fig. 2, which has no counterpart in the height image, is therefore, most probably caused by a repulsive interaction between the hydrophilic tip and the hydrophobic meshwork. In the first study in which the existence of a selective hydrophobic phase or meshwork was predicted, the authors have drawn a parallel between the hydrophobic meshwork in the NPC central channel and the lipid bilayer [8]. In their study, Ribbeck and Görlich point out that the hydrophobic barrier in the central channel might work analogously to a lipid bilayer. They also emphasise that the charge of a substrate, like the case for ions, has a big influence on whether it can be transported across the lipid bilayer or not. Considering this knowledge on lipid bilayers and the suggested analogy between the two structures, it may be hypothesised that separated pathways apart from the central channel are required for non-receptor mediated transport of ions and other hydrophilic molecules. Direct support for this hypothesis of a separate pathway for passive diffusion comes from a new publication by Naim et al. [14], in which the authors could show that passive and active transport are largely uncoupled. In a more recent publication, we have provided evidence that the pathway for ions and small macromolecules is indeed located at the periphery of NPCs. In the present work, we could show that the binding of an antibody against a peripheral NPC protein (Nup358) led to an increase in the electrical resistance of oocyte nuclei, whereas blocking of the central channel with importin $\beta 45$ 462 did not have any significant effect. This observation is again an indication for the existence of separate routes for receptor-mediated transport and passive diffusion. By comparing three antibodies with different targets at the periphery of the NPC, we were able to localise the cytoplasmic entrance to this peripheral route to the vicinity of the NPC protein Nup358 or, more specific, its internal repeat domain. This finding provides new perspectives for future investigations on the exact location of the peripheral routes. It still needs to be shown, however, whether these routes indeed 
coincide with the peripheral channels seen for the first time in 1992 by computational analysis of electronmicroscopic data [11].

\section{References}

1. Tran EJ, Wente SR (2006) Dynamic nuclear pore complexes: life on the edge. Cell 125:1041-1053

2. Stoffler D, Feja B, Fahrenkrog B, Walz J, Typke D, Aebi U (2003) Cryo-electron tomography provides novel insights into nuclear pore architecture: implications for nucleocytoplasmic transport. J Mol Biol 328:119-130

3. Gorlich D, Mattaj IW (1996) Nucleocytoplasmic transport. Science 271:1513-1518

4. Rout MP, Aitchison JD, Magnasco MO, Chait BT (2003) Virtual gating and nuclear transport: the hole picture. Trends Cell Biol 13:622-628

5. Pante N, Kann M (2002) Nuclear pore complex is able to transport macromolecules with diameters of about $39 \mathrm{~nm}$. Mol Biol Cell 13:425-434

6. Frey S, Richter RP, Gorlich D (2006) FG-rich repeats of nuclear pore proteins form a three-dimensional meshwork with hydrogellike properties. Science 314:815-817

7. Patel SS, Belmont BJ, Sante JM, Rexach MF (2007) Natively unfolded nucleoporins gate protein diffusion across the nuclear pore complex. Cell 129:83-96

8. Ribbeck K, Gorlich D (2001) Kinetic analysis of translocation through nuclear pore complexes. EMBO J 20:1320-1330

9. Ribbeck K, Gorlich D (2002) The permeability barrier of nuclear pore complexes appears to operate via hydrophobic exclusion. EMBO J 21:2664-2671

10. Bickel T, Bruinsma R (2002) The nuclear pore complex mystery and anomalous diffusion in reversible gels. Biophys J 83:3079-3087

11. Hinshaw JE, Carragher BO, Milligan RA (1992) Architecture and design of the nuclear pore complex. Cell 69:1133-1141

12. Danker T, Schillers H, Storck J, Shahin V, Kramer B, Wilhelmi M, Oberleithner H (1999) Nuclear hourglass technique: an approach that detects electrically open nuclear pores in Xenopus laevis oocyte. Proc Natl Acad Sci U S A 96:13530-13535

13. Kramer A, Ludwig Y, Shahin V, Oberleithner H (2007) A pathway separate from the central channel through the nuclear pore complex for inorganic ions and small macromolecules. J Biol Chem 28:28
14. Naim B, Brumfeld V, Kapon R, Kiss V, Nevo R, Reich Z (2006) Passive and facilitated transport in nuclear pore complexes are largely uncoupled. J Biol Chem 12:12

15. Shahin V, Danker T, Enss K, Ossig R, Oberleithner H (2001) Evidence for $\mathrm{Ca} 2+-$ and ATP-sensitive peripheral channels in nuclear pore complexes. FASEB J 15:1895-1901

16. Shahin V (2006) Route of glucocorticoid-induced macromolecules across the nuclear envelope as viewed by atomic force microscopy. Pflugers Arch 453:1-9

17. Boussu K, Van der Bruggen B, Volodin A, Snauwaert J, Van Haesendonck C, Vandecasteele C (2005) Roughness and hydrophobicity studies of nanofiltration membranes using different modes of AFM. J Colloid Interface Sci 286:632-638

18. Whangbo MH, Bar G, Brandsch R (1998) Qualitative relationships describing height and phase images of tapping mode atomic force microscopy. An application to micro-contact-printed patterned self-assembled monolayers. Appl Phys A Mater Sci Process 66:S1267-S1270

19. Schafer C, Ludwig Y, Shahin V, Kramer A, Carl P, Schillers H, Oberleithner H (2007) Ethanol alters access to the cell nucleus. Pflugers Arch 453:809-818

20. Kutay U, Bischoff FR, Kostka S, Kraft R, Gorlich D (1997) Export of importin alpha from the nucleus is mediated by a specific nuclear transport factor. Cell 90:1061-1071

21. Danker T, Mazzanti M, Tonini R, Rakowska A, Oberleithner H (1997) Using atomic force microscopy to investigate patchclamped nuclear membrane. Cell Biol Int 21:747-757

22. Joseph J, Liu ST, Jablonski SA, Yen TJ, Dasso M (2004) The RanGAP1-RanBP2 complex is essential for microtubule-kinetochore interactions in vivo. Curr Biol 14:611-617

23. Drummond SP, Wilson KL (2002) Interference with the cytoplasmic tail of gp210 disrupts "close apposition" of nuclear membranes and blocks nuclear pore dilation. J Cell Biol 158:53-62

24. Bernad R, van der Velde H, Fornerod M, Pickersgill H (2004) Nup358/RanBP2 attaches to the nuclear pore complex via association with Nup88 and Nup214/CAN and plays a supporting role in CRM1-mediated nuclear protein export. Mol Cell Biol 24:2373-2384

25. Vandamme N, Snauwaert J, Janssens E, Vandeweert E, Lievens P, Van Haesendonck C (2004) Visualization of gold clusters deposited on a dithiol self-assembled monolayer by tapping mode atomic force microscopy. Surf Sci 558:57-64

26. Dong R, Yu LE (2003) Investigation of surface changes of nanoparticles using TM-AFM phase imaging. Environ Sci Technol 37:2813-2819 